Adaptation, psychological processes 7

Advance care planning 247

Advanced glycation end products 127

Aging, biomedical research 420

-, postural control 157

-, ventral spinal artery 360

-, vertical gaze/convergence 177

Alzheimer's disease 110, 242

Anaemia 49

Aneurysm repair 349

Antiaging strategies 420

Anticoagulant 102

Antioxidant enzyme 57

Aortic aneurysm 349

- - screening 349

Apolipoproteins 206

Arterial morphology, rat spinal cord 360

Autologous transfusion 349

\section{Balance 135}

- confidence 373

- rehabilitation 87

- training 411

Behavioral genetics 22

Belgrade, dementia prevalence 260

Benign paroxysmal positional vertigo 82

Biological age 64

Biophysical measures, ESAP 330

Blood pressure 102, 223, 315

- - changes 182

- transfusion strategies 349

Body composition, Mexican elderly 366

- -, Venezuelan elders 223

Brain biopsy 235

- magnetic resonance imaging 200

Byzantine State, nursing homes, old 113

Capillary density 360

Cardiac surgery, quality of life 407

Cardiovascular risk factors 223

Cellular senescence 420

Charity 113

Cholesterol 206

Cognition 230

Cognitive abilities 35

- aging $5,7,12,17,28$

- change (12-year) 64

- decline 39,255

Competence assessment 330

C-reactive protein 216

- - cutoff level 216

Cross-domain variability 39
Death prediction 309

Dementia 39, 171

- prevalence 260

Depression 303

Diagnostic criteria, anaemia 49

Dizziness Handicap Inventory 82

Dual tasking 157

Dynamic balance 87

Elastic fiber 360

Elderly ambulatory women, fractures/death 309

-, anaemia 49

-, attitudes towards life 247

-, frail 411

- women, balance training 87

Electromyography, mental stress 383

Elevated plus-maze test 200

Emotion regulation 22

Emphysematous cystitis 259

Endovascular aneurysm repair 349

- stent grafting 349

Enteral nutrients, half-solid 417

Epley's manoeuvre 82

Everyday competence 291

Exercise 121, 373

Falls 303,411

-, fear 411

-, risk 373

Fasting 206

Field evaluation, cycled coupled movements, hand/foot 399

Fistula 259

Fracture prediction 309

Functional dependency 171

Gastroesophageal reflux 417

Gastroscopy 182

Gender, cognitive functioning 35

Gene expression, fasting effects 206

Glutathione peroxidase 193

Health survey, benign paroxysmal positional vertigo 82

Heart rate response, gastroscopy 182

Hip fracture 298

- -, complications 216

- -, infections 216

Homolateral hand/foot coordination 399

Hospital discharge 298
Hot-plate nociceptive tests, memory impairment 152

Human heart 127

- skin fibroblast 193

Hydrogen peroxide 193

Hyperosmolar dehydration 171

- syndromes, mortality 171

Institutionalised elderly people 96

- _ - , quality of care 96

Interventional recanalization, occluded peripheral arteries 393

Intraindividual variability $5,7,22,28,44$, 255

Japan, dementia, mortality effects 110

Ketamine 152

Latent change models, cognitive functioning 35

- growth/multilevel models, cognitive aging 12

Life expectancy 165

- orientation 247

Linoleic acid hydroperoxide 193

Lipid metabolism 206

Living will 247

Loneliness 291

Low bone mass 373

Low-vision aids 96

Masticatory muscle activity, acute mental stress 383

Medical assessment, aneurysm surgery 349

Memory 152

Mental stress 383

Mini-Mental State Examination 39

Mitochondrial dysfunction 187

- genetics 187

- metabolic competence 187

- structural dynamics 187

- therapeutics 187

Mitosis 265

'Multiple-Sit-to-Stand' Field Test 121

Negative affect 22

Nested latent growth curve analyses, processing speed 17

Neuronal noise 28

$\mathrm{N}$-nitrosobis(2-oxopropyl)amine 57 
Normative crying 322

Nursing home 165, 298

Ocular convergence 177

Office blood pressure 315

Off-target verbosity 291

Oldest old 39

Oxidative DNA damage 57

- stress 193

8-Oxo-2'-deoxyguanosine 57

Percutaneous endoscopic gastrostomy 417

Perinatal asphyxia 200

Peripheral arterial occlusive disease 393

Periventricular hyperintensities 242

Physical activity 135, 230

Physiological status, cognitive changes 64

Population survey, dementia 260

Postural control 157

Posture/gait abnormalities 303

Primary brain tumour 235

Processing fidelity 28

Psychosocial determinants, competence assessment 330

Puberty 265

Pulmonary function 230
Rat spinal cord, angioarchitecture 360

Regenerative biology 420

Reproductive hormones 265

Resuscitation preferences, living will 247

Saliva flow rate 383

Salivary cortisol 383

Scopolamine 152

Seasonal blood pressure changes, essential hypertension 315

Secondary task processing 157

Sensorimotor functioning 28

Sensory function 64

SF-36 82, 407

Shape aftereffect 142

- perception 142

Short-term vs. long-term changes, processing speed 17

Smoking 230

Social behavior, perinatal asphyxia, aging rats 200

Sociodemographics, competence assessment 330

Somatotype 223

Strategies, engineered negligible senescence 420
Strength 135

Stroke 102

- outcome 102

Stroke/aging, crying 322

Subjective well-being 291

Successful aging 330

Synaptic mitochondria 187

Telomere aging 420

Theories, aging 7, 44

Three-compartment model, body composition 366

Triglyceride 206

Uncontrolled crying 322

Vascular dementia 110

- factors, Alzheimer's disease 242

Ventral spinal artery 360

Vertical eye movement 177

Visual disability 96

Weibull model, survival prediction, Chinese nursing home residents 165

White matter changes 242 